\title{
Prática de atividade física no tempo livre entre adultos brasileiros durante o período de 2011 a 2019
}

\author{
Practice of free-time physical activity among brazilian adults from 2011 to 2019 \\ Práctica de actividad física en el tiempo libre en adultos brasileños durante el periodo de 2011 a
}

\section{Resumo}

Objetivo: Descrever a prática de atividade física no tempo livre entre adultos brasileiros durante o período de 2011 a 2019. Método: Estudo epidemiológico ecológico descritivo, a partir de dados secundários do Sistema de Vigilância de Fatores de Risco e Proteção para Doenças Crônicas por Inquérito Telefônico (VIGITEL), no período de 2011 a 2019. A população foi constituída por adultos ( $\geq 18$ anos de idade) brasileiros que participaram do VIGITEL. As variáveis de interesse foram: ano, sexo, faixa etária, escolaridade, região e capital. Resultados: Observou-se que a prática de atividade física no tempo livre foi maior entre os anos de 2011 a 2015, entre os adultos do sexo masculino (45,9\%), na faixa etária de 18 a 24 anos $(48,1 \%)$ e que possuíam nove ou mais anos de estudo. No período analisado, as regiões Sul $(39,6 \%)$, Centro-Oeste $(39,6 \%)$ e Norte $(39,2 \%)$ apresentaram maior frequência da prática de atividade física no tempo livre, sendo Florianópolis, Distrito Federal e Palmas as capitais que apresentaram maior índice de atividade física. Conclusão: Houve maior prevalência da prática de atividade física nas cidades de Florianópolis, Palmas e Distrito Federal, e nas regiões Sul, Centro Oeste e Norte. Homens, adultos jovens e indivíduos com maior nível de escolaridade apresentaram melhores índices da prática de atividade física no lazer.

Palavras-chave: Atividade física; Atividades de lazer; Adultos; Epidemiologia; Inquéritos populacionais.

\begin{abstract}
Objective: Describe the practice of free time physical activity among adults in Brazil from 2011 to 2019. Materials and Methods: Descriptive ecological epidemiological study, using secondary data from the Risk and Protection Factors Surveillance System for Chronic Diseases by Telephone Survey in the period from 2011 to 2019. The population consisted of adults ( $\geq 18$ years old). The following variables were chosen: year, sex, capital, region, age group and education. Results: In the period analyzed, the South (39.6\%), Midwest (39.6\%) and North (39.2\%) regions had the highest average of physical activity. Florianópolis, Distrito Federal and Palmas were the capitals that presented the highest index of physical activity. It was observed that the practice of physical activity during leisure was higher in males $(45.9 \%)$, in the age group of 18 to 24 years $(48.1 \%)$ and in individuals who had 9 or more years of study. Conclusion: Men, young adults and individuals with a higher level of education had better rates of physical activity during leisure time. Higher prevalence of physical activity in the cities of Florianópolis, Palmas and the Federal District and in the South, Midwest and North regions.
\end{abstract}

Keywords: Physical activity; Leisure activities; Adults; Epidemiology; Demographic surveys.

\section{Resumen}

Objetivo: Describir la práctica de actividad física en el tiempo libre en adultos en Brasil de 2011 a 2019. Materiales y Métodos: Estudio epidemiológico ecológico descriptivo, con base en datos secundarios del Sistema de Vigilancia de 
Factores de Riesgo y Protección de Enfermedades Crónicas por Encuesta Telefónica (VGITEL ), de 2011 a 2019. La población estuvo constituida por adultos brasileños ( $\geq 18$ años) que participaron en VIGITEL. Las variables de interés fueron: año, sexo, grupo de edad, educación, región y capital. Resultados: Se observó que la práctica de actividad física en el tiempo libre fue mayor entre los años 2011 a 2015 , en hombres (45,9\%), de 18 a 24 años (48,1\%) y en individuos que tenían 9 o más años de escolaridad. En el período analizado, las regiones Sur (39,6\%), Medio Oeste $(39,6 \%)$ y Norte $(39,2 \%)$ tuvieron el mayor média de actividad física en tiempo libre, siendo Florianópolis, Distrito Federal y Palmas las capitales que presentaron la mayor tasa de actividad física. Conclusión: Se observó una mayor prevalencia de actividad física en las ciudades de Florianópolis, Palmas y Distrito Federal, y en las regiones Sur, Medio Oeste y Norte. Los hombres, los adultos jóvenes y las personas con un mayor nivel de educación tuvieron mejores tasas de actividad física en el tiempo libre.

Palabras clave: Actividad física; Actividades recreativas; Adultos; Epidemiología; Encuestas de población.

\section{Introdução}

Devido ao processo de globalização, urbanização e avanços tecnológicos ao longo dos anos, a sociedade moderna apresenta um novo perfil de adoecimento da população. Mudanças no estilo de vida dos indivíduos, incluindo a redução das práticas de atividade física e aumento do tempo com comportamento sedentário também são considerados fatores que influenciam neste perfil (Bull, Goenka, Lambert, \& Pratt, 2017).

Atualmente as doenças crônicas não transmissíveis (DCNT) representam um dos principais problemas de saúde pública, as prevalentes causas de morte no mundo, e têm como um dos fatores de risco mais relevante à inatividade física (Booth, Roberts, Thyfault, Ruegsegger, \& Toedebusch, 2017). Segundo a Organização Mundial da Saúde (OMS) pessoas que são consideradas insuficientemente ativas têm um risco de morte de $20 \%$ a $30 \%$ maior quando comparadas com pessoas suficientemente ativas (World Health Organization, 2018).

A redução dos níveis de atividade física e o aumento do tempo gasto em atividades sedentárias pode fazer com que a população desenvolva doenças cardiovasculares, respiratórias, metabólicas, musculoesqueléticas, câncer e depressão (Patterson et al., 2018). Por outro lado, a realização de atividade física é considerada como uma estratégia para a prevenção e gestão de doenças crônicas e ainda para a melhora da saúde mental, reduzindo o risco de depressão e deficiência cognitiva e melhorando e elevando a autoestima (Villalobos et al., 2019).

No Brasil, a inatividade física é responsável de 3\% a 5\% de todas as principais DCNT e 5,31\% de todas as causas de mortalidade, variando de 5,82\% na região sudeste a 2,83\% na região sul (Silva et al., 2017). De acordo com a OMS, um em cada quatro adultos no mundo não atinge os níveis globais recomendados de atividade física, o que reflete nos cinco milhões de mortes por ano que poderiam ser evitadas se a população global fosse mais ativa (World Health Organization, 2018). O cumprimento ou não das recomendações sobre a prática da atividade física é uma variável investigada em diversos estudos e em diferentes países. Por exemplo, no Brasil, a taxa de sedentarismo aumentou desde 2002 em mais de 15\%, e dados indicam que mais de 47\% dos brasileiros em 2016 eram sedentários (Guthold, Stevens, Riley, \& Bull, 2018).

A prática da atividade física é descrita como um dos principais fatores comportamentais de proteção das Doenças Crônicas, pela redução do risco de mortalidade por todas as causas, prevenção de doenças cardiovasculares (DCV) e diabetes, melhoria dos níveis de lipídios, redução da hipertensão e dos riscos de câncer de mama e cólon (Bull, Goenka, Lambert, \& Pratt, 2017). De acordo com esses autores, ainda tem efeitos positivos na saúde mental, retarda o início da demência e pode ajudar na manutenção de um peso saudável.

Outro fato relevante diz respeito aos danos causados às sociedades pelos custos indiretos da inatividade física, entre os quais estão às perdas de produtividade, devido à morbidade e mortalidade prematura, maior uso de combustíveis fósseis que poluem o ar e estradas mais congestionadas e inseguras (Guthold, Stevens, Riley, \& Bull, 2018).

A OMS define atividade física como qualquer movimento corporal produzido pelos músculos esqueléticos que requer gasto de energia e se refere a todos os movimentos durante o tempo de lazer, transporte para ir e voltar de lugares, ou como 
parte do trabalho de uma pessoa (World Health Organization, 2018).

A principal recomendação da OMS é que os indivíduos adultos devem praticar pelo menos de 150 minutos a 300 minutos por semana de intensidade moderada, ou 75 minutos a 150 minutos por semana de atividade aeróbia de intensidade moderada e vigorosa, além de fazer atividades de fortalecimento muscular em um ou mais dias por semana (World Health Organization, 2018). Entretanto destaca-se que em relação ao tipo da atividade física, preferencialmente deve ser a aeróbica, podendo ser realizada com diferentes graus de esforço, diferentes tempos de duração e ainda incluir diversos tipos de atividades em variados tipos de ambientes como atividades de esportes, recreação, caminhadas, trote, corrida, ciclismo e natação (Bull et al., 2020).

Desde o ano de 2006, o Sistema de Vigilância de Fatores de Risco e Proteção para Doenças Crônicas por Inquérito Telefônico (VIGITEL), do Ministério da Saúde, vem consolidando e disponibilizando dados nacionais sobre vários indicadores de saúde, dentre os quais está a prática de atividade física em adultos maiores de 18 anos.

A pesquisa VIGITEL é realizada pela Secretaria de Vigilância em Saúde (SVS) do Ministério da Saúde, com maiores de 18 anos, nas 26 capitais brasileiras e no Distrito Federal e consiste em um estudo transversal de base populacional que avalia anualmente diversos assuntos relacionados à saúde, por meio de entrevistas telefônicas.

Considerando a relevância do tema e a escassez de publicação sobre a temática e metodologia aplicada nesta pesquisa no período analisado, este estudo tem como objetivo de pesquisa descrever a prática de atividade física no tempo livre entre adultos do Brasil no período de 2011 a 2019.

\section{Metodologia}

Estudo epidemiológico ecológico descritivo sobre a prática da atividade física no tempo livre na população adulta brasileira ( $\geq 18$ anos de idade), entre os anos de 2011 e 2019. Os dados foram obtidos a partir das informações publicadas pelos relatórios anuais do Sistema de Vigilância de Fatores de Risco e Proteção para Doenças Crônicas (VIGITEL).

Foram utilizadas as informações registradas pelo VIGITEL no período de 2011 a 2019 sobre prática de atividade física no tempo livre em pessoas com idade igual ou maior de 18 anos, nas capitais do Brasil e no Distrito Federal. Apesar do VIGITEL disponibilizar informações desde 2006, o período de estudo foi a partir do ano de 2011, porque houve mudança na metodologia de avaliação deste indicador neste ano, acompanhando as mudanças nas recomendações internacionais da OMS. Essa mudança foi referente à forma de cálculo relacionado ao volume de prática de atividade física recomendada no tempo livre. O mesmo deixou de estipular um número mínimo de dias na semana para a prática da atividade física, e passou a usar o número de indivíduos que praticam pelo menos 150 minutos semanais de atividade física de intensidade leve ou moderada, ou pelo menos 75 minutos semanais de atividade física de intensidade vigorosa, dividido pelo número de indivíduos entrevistados (Ministério da Saúde, 2012).

O processo amostral utilizado no VIGITEL foi do tipo probabilístico e foi constituído por sorteio de 5.000 residências com linha telefônica fixa por cidade, seguido de sorteio de um morador com idade $>18$ anos por domicílio até se obter o número mínimo de 2.000 entrevistas por cidade. O número de entrevistas completas realizadas pelo VIGITEL a cada ano foi: 54.144 em 2011, 45.448 em 2012, 52.929 em 2013, 40.853 em 2014, 54.174 em 2015, 53.210 em 2016, 53.034 em 2017, 52.395 em 2018 e 52.443 entrevistas em 2019, totalizando 458.630 no período analisado. Os dados foram acessados entre março e maio de 2021.

As informações sobre a prática de atividade física no tempo livre ou de lazer foram obtidas a partir das seguintes questões do VIGITEL: "Nos últimos três meses, o(a) Sr.(a) praticou algum tipo de exercício físico ou esporte?", "Qual o tipo principal de exercício físico ou esporte que o(a) Sr.(a) praticou?", “O(a) Sr.(a) pratica o exercício pelo menos uma vez por semana?", "Quantos dias por semana o(a) Sr.(a) costuma praticar exercício físico ou esporte?" e "No dia que o(a) Sr.(a) pratica 
exercício ou esporte, quanto tempo dura essa atividade?".

O percentual de adultos que praticam atividades físicas no tempo livre equivalente a pelo menos 150 minutos de atividade de intensidade moderada por semana foram calculados dividindo o número de indivíduos que praticam pelo menos 150 minutos semanais de atividade física de intensidade moderada ou pelo menos 75 minutos semanais de atividade física de intensidade vigorosa pelo número de indivíduos entrevistados. As variáveis selecionadas para análise foram as já existentes no VIGITEL como, ano, sexo, região, capital, faixa etária e escolaridade.

Foi calculado o percentual médio da prática da atividade física no tempo livre de cada região para análise comparativa entre as mesmas. As informações relacionadas às variáveis de interesse foram analisadas por meio da frequência absoluta e relativa e apresentadas em tabelas e gráficos. Para auxiliar a análise dos dados foi utiliza o programa Microsoft Excel 2016.

O estudo utilizou dados secundários de domínio público, dessa forma, não apresenta implicações éticas e morais e dispensa a autorização do comitê de ética em pesquisa, estando assim de acordo com as normas da Resolução 466/12 do Conselho Nacional de Ética em Pesquisa.

\section{Resultados}

Observa-se um aumento da prática de atividade física em todas as regiões entre os anos de 2011 a 2015 . A partir de 2016, observa-se a ocorrência de aumento e/ou redução nas médias em todas as regiões até 2019. As regiões Sul (39, 6\%), Centro Oeste $(39,6 \%)$ e Norte $(39,2 \%)$ apresentaram maior média da prática da atividade física e as regiões Nordeste $(36,1 \%)$ e Sudeste (36,9\%) menor média no período analisado. A Tabela 1 descreve a prevalência de adultos que praticavam atividade física suficiente no tempo livre, nas capitais dos estados das regiões brasileiras no período analisado.

Tabela 1. Prevalência de atividade física suficiente no tempo livre entre adultos nas capitais dos estados das regiões brasileiras no período de 2011 a 2019. Brasil, 2021.

\begin{tabular}{|c|c|c|c|c|c|c|c|c|c|}
\hline Ano & 2011 & 2012 & 2013 & 2014 & 2015 & 2016 & 2017 & 2018 & 2019 \\
\hline Regiões & $\%$ & $\%$ & $\%$ & $\%$ & $\%$ & $\%$ & $\%$ & $\%$ & $\%$ \\
\hline Nordeste & 26,7 & 34,1 & 34,7 & 35,4 & 36,6 & 38,1 & 37,8 & 41,1 & 40,8 \\
\hline Norte & 31,8 & 36,3 & 36,2 & 39,3 & 42,4 & 40,0 & 42,0 & 43,7 & 42,2 \\
\hline Centro-oeste & 30,7 & 36,6 & 38,8 & 38,7 & 44,0 & 41,0 & 42,8 & 42,0 & 41,4 \\
\hline Sul & 36,0 & 38,4 & 39,7 & 40,0 & 41,0 & 38,2 & 40,1 & 41,2 & 41,3 \\
\hline Sudoeste & 32,1 & 35,3 & 35,0 & 37,3 & 39,1 & 39,2 & 37,5 & 38,0 & 39,0 \\
\hline
\end{tabular}

Fonte: VIGITEL- Sistema de Vigilância de Fatores de Risco e Proteção para Doenças Crônicas por Inquérito Telefônico.

A prevalência da prática de atividade física no tempo livre nas capitais dos estados brasileiros e Distrito Federal variou entre 31,5\% em 2011 a 40,9\% em 2019, ocorrendo um aumento de 29,8\% no período analisado.

$\mathrm{Na}$ Tabela 2, estão os dados referentes à prática de atividade física no tempo livre nas capitais dos estados brasileiros $\mathrm{e}$ Distrito Federal, onde pode-se notar que no ano de 2011, a frequência de adultos que praticavam atividade física variou entre 26,3\% em Porto Velho e 41,4\% em Florianópolis.

Florianópolis foi a capital com maior prevalência da prática de atividade física no tempo livre nos anos de 2012 (43,1\%), 2013 (43,9\%) e 2014 (47,1\%), já São Paulo apresentou a menor prevalência neste mesmo período: 2012 (27,9\%), 2013 (28,0\%), e 2014 (30,4\%). O Distrito Federal assume a maior prevalência nos anos de 2015 (52,1\%), 2016 (46,2\%) e 2017 (49,6\%). Em 2018 e 2019 a capital Palmas tem maior prevalência com 47,0\% e 40,9\% respectivamente. 
Tabela 2. Prevalência da prática de atividade física no tempo livre entre adultos das capitais dos estados brasileiros e Distrito Federal no período de 2011 a 2019. Brasil, 2021

\begin{tabular}{|c|c|c|c|c|c|c|c|c|c|}
\hline Ano & 2011 & 2012 & 2013 & 2014 & 2015 & 2016 & 2017 & 2018 & 2019 \\
\hline Capital & $\%$ & $\%$ & $\%$ & $\%$ & $\%$ & $\%$ & $\%$ & $\%$ & $\%$ \\
\hline Aracaju & 30,3 & 38,5 & 37,3 & 37,2 & 43,6 & 40,2 & 40,3 & 44,3 & 41,9 \\
\hline Belém & 34,2 & 38,3 & 36,2 & 36,6 & 41,7 & 39,3 & 41,3 & 42,7 & 43,2 \\
\hline Belo Horizonte & 35,0 & 36,4 & 35,9 & 39,9 & 38,8 & 40,7 & 38,9 & 41,2 & 39,7 \\
\hline Boa Vista & 33,0 & 35,9 & 40,2 & 41,0 & 45,9 & 40,2 & 40,7 & 41,6 & 39,7 \\
\hline Campo Grande & 30,7 & 36,3 & 40,0 & 38,3 & 39,9 & 38,9 & 40 & 40,1 & 39,5 \\
\hline Cuiabá & 29,4 & 34,7 & 36,5 & 39,9 & 41,0 & 37,7 & 40,6 & 39,9 & 38,3 \\
\hline Curitiba & 33,2 & 35,1 & 37,4 & 36,8 & 41,0 & 39,3 & 41,6 & 41,3 & 41,0 \\
\hline Distrito Federal & 32,8 & 38,7 & 41,5 & 37,4 & 52,1 & 46,2 & 49,6 & 45,6 & 47,1 \\
\hline Florianópolis & 41,4 & 43,1 & 43,9 & 47,1 & 47,6 & 42,1 & 43,6 & 46,8 & 45,2 \\
\hline Fortaleza & 30,0 & 32,7 & 35,4 & 37,5 & 38,1 & 38,9 & 37,2 & 42,4 & 40,3 \\
\hline Goiânia & 29,9 & 36,7 & 37,2 & 39,2 & 42,8 & 41,5 & 40,7 & 42,4 & 41,0 \\
\hline João Pessoa & 30,2 & 34,7 & 31,6 & 35,7 & 34,1 & 35,7 & 34,4 & 39,3 & 40,1 \\
\hline Macapá & 32,5 & 37,5 & 36,2 & 39,2 & 44,6 & 43,6 & 45,5 & 46,8 & 44,3 \\
\hline Maceió & 29,2 & 34,9 & 35,9 & 32,8 & 34,4 & 38,1 & 38,1 & 39,9 & 39,9 \\
\hline Manaus & 32,6 & 37,8 & 33,1 & 33,6 & 39,7 & 37,2 & 38,4 & 40,1 & 37,1 \\
\hline Natal & 31,8 & 35,0 & 38,2 & 40,4 & 39,7 & 39,3 & 38,8 & 42,5 & 45,4 \\
\hline Palmas & 33,8 & 38,1 & 40,2 & 41,4 & 46,6 & 44,9 & 45,9 & 47,0 & 49,9 \\
\hline Porto Alegre & 33,6 & 37,0 & 37,6 & 35,9 & 34,5 & 33,4 & 35,3 & 35,6 & 37,7 \\
\hline Porto Velho & 26,3 & 34,5 & 31,6 & 41,2 & 39,1 & 38,3 & 41,3 & 44,7 & 37,1 \\
\hline Recife & 28,7 & 31,7 & 34,6 & 31,6 & 31,7 & 37,1 & 35,2 & 37,3 & 35,6 \\
\hline Rio Branco & 30,1 & 32,6 & 36,2 & 42,5 & 39,4 & 37,1 & 40,3 & 42,6 & 44,6 \\
\hline Rio de Janeiro & 30,0 & 34,0 & 33,0 & 37,6 & 41,0 & 38,9 & 36,9 & 34,8 & 37,8 \\
\hline Salvador & 28,3 & 32,5 & 33,6 & 32,4 & 34,6 & 38,0 & 36,3 & 42,0 & 41,3 \\
\hline São Luís & 29,4 & 33,4 & 30,9 & 36,9 & 37,9 & 37,9 & 41,9 & 40,4 & 37,9 \\
\hline São Paulo & 27,5 & 27,9 & 28,0 & 30,4 & 30,7 & 32,4 & 29,9 & 31,0 & 34,6 \\
\hline Teresina & 31,9 & 33,7 & 34,6 & 34,5 & 35,3 & 38,1 & 37,5 & 42,6 & 44,3 \\
\hline Vitória & 36,1 & 43,1 & 43,0 & 41,3 & 46,2 & 45,1 & 44,4 & 45,2 & 44,2 \\
\hline Prevalência total & 31,5 & 36,1 & 36,9 & 38,1 & 40,6 & 39,3 & 40,4 & 41,2 & 40,9 \\
\hline
\end{tabular}

Fonte: VIGITEL- Sistema de Vigilância de Fatores de Risco e Proteção para Doenças Crônicas por Inquérito Telefônico.

Com relação à prática de atividade física no tempo livre segundo o sexo, os homens apresentaram prevalências maiores em todos os anos, variando de 41,4\% em 2011 a 48,2\% em 2019. A prevalência nas mulheres variou de 24,0\% em 2011 e 28,0\% em 2019 (Figura 2). Comparando a média da prática de atividade física no tempo livre entre o sexo masculino $(45,9 \%)$ e o sexo feminino $(31,7 \%)$, verifica-se uma razão de 1,45 .

Na Tabela 3 está descrito as prevalências da prática de atividade física no tempo livre entre adultos, segundo faixa etária e escolaridade. Para todas as faixas etárias houve aumento da prática de atividade física no período analisado. Sendo a faixa etária de 18 a 24 anos, a que apresentou maior prevalência $(48,1 \%)$, com tendência a redução da prática de atividade física no tempo livre com o aumento da idade, chegando a uma frequência de 23,1\% entre as pessoas com 65 ou mais anos.

Com relação à escolaridade, houve aumento da prática de atividade física no tempo livre no período analisado para as pessoas que tinham entre 9 a 11 anos e 12 ou mais anos de estudo. Nota-se que nesse período a frequência dessa prática tendeu a diminuir com o aumento da idade e aumentou com o nível de escolaridade. 
Tabela 3. Prevalência da prática de atividade física no tempo livre entre adultos das capitais dos estados brasileiros e Distrito Federal por sexo, faixa etária e escolaridade no período de 2011 a 2019. Brasil, 2021

\begin{tabular}{|c|c|c|c|c|c|c|c|c|c|c|}
\hline Ano & 2011 & 2012 & 2013 & 2014 & 2015 & 2016 & 2017 & 2018 & 2019 & Média \\
\hline Variáveis & $\%$ & $\%$ & $\%$ & $\%$ & $\%$ & $\%$ & $\%$ & $\%$ & $\%$ & $\%$ \\
\hline \multicolumn{11}{|l|}{ Sexo } \\
\hline Masculino & 41,4 & 44,2 & 44,3 & 45 & 47,6 & 47,5 & 46,5 & 48,4 & 48,2 & 41,4 \\
\hline Feminino & 24,0 & 29,0 & 30,4 & 32,1 & 34,6 & 32,2 & 34,0 & 34,9 & 34,6 & 24,0 \\
\hline \multicolumn{11}{|c|}{ Faixa etária } \\
\hline $18-24$ & 40,5 & 47,6 & 49,7 & 50,0 & 51,4 & 52,2 & 49,1 & 50,6 & 49,4 & 48,1 \\
\hline $25-34$ & 32,1 & 39,1 & 39,3 & 41,5 & 45,2 & 46,0 & 44,2 & 45,5 & 48,5 & 40,8 \\
\hline 35- 44 & 26,4 & 31,0 & 29,6 & 31,2 & 36,4 & 35,7 & 33,8 & 36,0 & 36,8 & 33,0 \\
\hline $45-54$ & 26,2 & 25,8 & 27,3 & 30,1 & 30,5 & 30,4 & 33,7 & 32,6 & 34,6 & 30,1 \\
\hline $55-64$ & 24,7 & 25,2 & 26,6 & 28,4 & 29,1 & 29,7 & 30,0 & 32,4 & 31,5 & 24,7 \\
\hline 65 e mais & 22,2 & 23,6 & 22,3 & 22,8 & 23,5 & 22,3 & 23,3 & 24,4 & 24,4 & 23,1 \\
\hline \multicolumn{11}{|c|}{ Escolaridade } \\
\hline $0-8$ & 24,0 & 21,6 & 22,0 & 22,9 & 25,4 & 24,5 & 23,3 & 24,6 & 25,8 & 23,9 \\
\hline $9-11$ & 35,1 & 37,1 & 37,2 & 38,5 & 40,1 & 40,4 & 39,7 & 40,4 & 39,5 & 38,7 \\
\hline $12 \mathrm{e}$ mais & 42,2 & 45,4 & 45,4 & 47,8 & 49,6 & 47,9 & 47,0 & 48,1 & 50,0 & 47,0 \\
\hline
\end{tabular}

Fonte: VIGITEL- Sistema de Vigilância de Fatores de Risco e Proteção para Doenças Crônicas por Inquérito Telefônico.

\section{Discussão}

A partir da coleta sistemática de dados realizados pelo VIGITEL, entre os anos de 2011 e 2019, em uma amostra representativa da população adulta das 26 capitais dos estados brasileiros e do Distrito Federal foi possível analisar a evolução da prática de atividade física no tempo livre (AFTL) no país. O estudo apontou um aumento na AFTL em todas as regiões do país no período analisado. Este achado merece destaque considerando que a prática de atividade física confere maiores benefícios à saúde, incluindo melhorias no bem-estar físico, psicológico e social e redução da morbimortalidade por DCNT, especialmente as cardiovasculares (Silva, Souza, Rossi Filho, Silva, \& Rigoni, 2017).

Outra questão importante concerne às diferenças da AFTL entre as regiões encontradas neste estudo. Ainda que estas diferenças sejam pequenas, é sabido que questões culturais, econômicas, sociais, de segurança, disponibilidade de espaços e ainda, nível educacional e a percepção que a população tem das condições do bairro de cada região, são fatores que podem interferir no desenvolvimento da prática atividade física em áreas livres (Wendt, Carvalho, Silva, \& Mielke, 2019).

A Pesquisa Nacional de Saúde realizada em 2019 verificou que menor proporção de mulheres praticavam exercício físico no lazer $(24,3 \%)$ ocorreu na região Norte, enquanto que foi a região Sudeste que apresentou a maior proporção de homens (36,1\%) (Instituto Brasileiro de Geografia e Estatística [IBGE], 2020). O crescimento desordenado do meio urbano de algumas capitais brasileiras pode ocasionar diversos problemas ambientais e estruturais que funcionam como barreiras da AFTL, como caminhadas, corridas e ciclismo (Pitanga et al., 2019).

Os resultados deste estudo mostram que ao longo do período analisado a maioria das capitais apresentou aumento das prevalências AFTL, com destaque para as capitais Florianópolis, Distrito Federal e Palmas. Para o entendimento das diferenças das prevalências de AFTL entre as capitais, não podem ser desconsiderados os condicionantes ambientais do meio físico, decorrentes do processo específico de urbanização de cada localidade. Estudo multicêntrico realizado com 8.023 participantes 
de seis diferentes cidades do Brasil verificou que a percepção favorável do ambiente está associado ao aumento da prática atividade física no tempo livre (Chor et al., 2016).

Com relação ao sexo que mais pratica AFTL, constatou-se uma significativa predominância do sexo masculino, sendo este padrão também observado num estudo realizado com 116.982 adultos de cinco países da América do Sul, no qual constatou-se que a atividade física foi maior entre os homens do que entre as mulheres em todos os países, exceto na Argentina (Werneck et al., 2019).

Dados da Pesquisa Nacional de Saúde realizada em 2019 confirmam que homens com 18 anos ou mais praticaram o nível recomendado de AFTL, quando comparado com as mulheres (Instituto Brasileiro de Geografia e Estatística [IBGE], 2020). Resultados de um estudo multicêntrico também evidenciaram que no lazer, as mulheres e pessoas com menor escolaridade e menor renda média per capita são as menos ativas (Chor et al., 2016). As mulheres são menos propensas do que os homens a serem fisicamente ativas e mais propensas a reduzir sua atividade física à medida que envelhecem (Jones, Leeman, \& Evenson, 2020).

Esta diferença da prevalência de AFTL entre homens e mulheres pode ser explicada pelas diferenças de gênero, portanto como um fenômeno social com base na construção de papeis em um contexto social mais amplo (Oliveira, et al., 2014). Para esses autores, menor frequência de AFTL entre as mulheres tem relação com a realidade de suas vidas cotidianas e, particularmente com as responsabilidades das mulheres com o trabalho doméstico e com a família, e consequentemente com menor tempo para dedicar-se a esta prática.

Outro ponto que cabe ser destacado é que mulheres que são mães, que tem desvantagens socioeconômicas e residem em bairros mais populares, apresentam risco mais elevado de inatividade física e altos níveis de tempo de tela (Morris, Arundell, Cleland, \& Teychenne, 2020). Já com relação aos homens a maior prevalência de AFTL pode ser justificada pela cultura quanto aos motivos que levam os indivíduos do sexo masculino, desde a infância, a serem incentivados a participar de atividades físicas, principalmente as atividades coletivas, esportes e outras atividades mais vigorosas com maior gasto de energia (Dumith et al., 2016).

É importante salientar que se faz necessária à redução das iniquidades relacionadas ao sexo feminino, compreendendo e lidando com essas barreiras por meio de ações culturalmente sensíveis para apoiar a mudança de comportamento e maior prevalência de participação delas nas AFTL (Guthold, Stevens, Riley, \& Bull, 2018).

Destacou-se neste estudo a baixa prevalência da AFTL entre as pessoas com mais 55 anos ou mais, comparado aos adultos mais jovens (menos de 25 anos de idade). Corroborando este resultado, um estudo transversal realizado com dados coletados na Pesquisa Nacional de Saúde (PNS) em 2013, observou que a faixa etária predominante na prática de atividade física, também foi de 18 a 24 anos (23,1\%) (Wendt, Carvalho, Silva, \& Mielke, 2019). O predomínio da população jovem neste contexto pode ser explicado devido aos jovens se sentirem mais motivados, terem mais tempo livre, e apresentarem desejo de melhorar a condição física e estética, além de melhorar a saúde mental e qualidade de vida (Instituto Brasileiro de Geografia e Estatística [IBGE], 2020).

A faixa etária com 65 anos ou mais apresentou a menor prevalência de AFTL neste estudo. Embora atividades físicas sejam importantes para todas as idades, elas são especialmente fundamentais para os indivíduos idosos, pois atuam como fator de proteção para DCNT. Além de promover o aumento da massa muscular, a AFTL melhora o equilíbrio e a marcha, atua na melhora da autoestima e da autoconfiança e adia o declínio funcional que ocorre no processo de envelhecimento (World Health Organization, 2018).

Segundo a OMS, é recomendado que os indivíduos idosos com 65 anos ou mais realizem atividade física de intensidade moderada, preferencialmente em grupos, com período mínimo de três vezes na semana e a intensidade da atividade é de acordo com as condições de saúde de cada pessoa (Bull et al., 2020). 
De acordo com as projeções do Instituto Brasileiro de Geografia e Estatística- IBGE, em 2060 o Brasil terá um quarto da população na terceira idade, equivalente a 58,2 milhões de pessoas, sendo a expectativa de vida ao nascer será de 77,9 anos para homens e 84,2 para as mulheres, contudo, haverá um crescimento de doenças crônicas degenerativas, com o avançar da idade (Instituto Brasileiro de Geografia e Estatística [IBGE], 2020). Diante de tais considerações é fundamental que o país amplie políticas públicas específicas para esta população, incluindo apoio para a prática diária de atividade física.

Outro aspecto importante analisado neste estudo se refere à relação da AFTL com a escolaridade. Observou-se que a frequência desta prática predominava principalmente entre as pessoas com mais anos de estudo. Indivíduos com nove ou mais anos de estudo relataram maior frequência da prática da atividade física do que quem tinha de 0 a 8 anos de estudo. De acordo com a Pesquisa Nacional por Amostra de Domicílios (PNAD) a chance de o indivíduo praticar atividade física aumenta em 271,9\% quando o trabalhador possui 12 a 14 anos de estudo, e a chance aumenta para 471,9\% para aqueles com grau de escolaridade de 15 anos ou mais (Instituto Brasileiro de Geografia e Estatística - IBGE (2020).

Considerando a correlação existente entre escolaridade e renda e entre outras condições socioeconômicas da população, no que diz respeito à moradia, acesso a possiblidades, facilidades e equipamentos, a locais públicos e privados adequados e seguros, a baixa escolaridade justifica a menor frequência da prática da AFTL. Baseado em tais considerações é importante à compreensão de que a prática da atividade física no tempo livre faz parte de um processo complexo e multidimensional e que não depende apenas da vontade e esforço do indivíduo (Carvalho, 2019; Moreno-Llamas, GarcíaMayor \& De la Cruz-Sánchez, 2020).

Considera-se que a classe social é um fator relevante para a baixa atividade física, principalmente com a existência de mais barreiras nas classes sociais mais baixas, tais como custos econômicos, maior frequência de deficiência ou doença e risco de lesões (Moreno-Llamas, García-Mayor \& De la Cruz-Sánchez, 2020). Dessa forma é plausível alertar para a necessidade de políticas públicas e programas de investimento em espaços que incentivem a prática de atividade física acessíveis à população de baixa escolaridade e renda.

Vale ressaltar ainda que é necessário ponderar a implicação de outros aspectos intervenientes tais como a urbanização, segurança, instalações e programas públicos, condições de saúde, renda, ocupação, escolaridade, o que implica a necessidade do envolvimento de vários setores, como Saúde, Educação, Transporte, Planejamento Urbano, entre outros (Carvalho, 2019). Essas considerações evidenciam que os profissionais de saúde precisam estar cientes de seu potencial para mediar e incentivar a prática da atividade física no tempo livre, não só entre os doentes, mas também na população como um todo, orientando sobre a forma de executá-la, enfatizando os seus benefícios e ajudando a enfrentar e solucionar as barreiras identificadas.

Apesar das informações sobre atividade física no tempo livre serem valiosas, e considerando que os dados analisados neste estudo se referem somente à atividade física no tempo livre ou de lazer, as mesmas não devem ser consideradas para definir se a população é ativa ou sedentária, pois esses comportamentos incluem outros domínios, como atividades desenvolvidas no trabalho, no deslocamento e no ambiente doméstico, que não foram aqui consideradas.

Dentre os limites do estudo, cita-se a utilização de dados secundários avaliados por meio de informação da AFTL autorreferida, que sugere uma possível superestimação das informações. Além disso, o fato de abordar apenas um dos domínios, o que impede uma avaliação da atividade física de uma forma mais ampla.

\section{Conclusão}

Os resultados apontam um aumento na prática de atividade física no lazer entre adultos nas capitais de estados do país e no Distrito Federal, com maior prevalência nas cidades de Florianópolis, Distrito Federal e Palmas e em menor prevalência na cidade de São Paulo. As regiões Sul, Centro Oeste e Norte apresentaram os melhores índices de AFTL e as regiões Nordeste e Sudeste os menores índices no período analisado. Esse aumento foi especialmente relevante entre os homens jovens na faixa 
etária de 18 a 24 anos e aqueles indivíduos com maior nível de escolaridade.

Os resultados evidenciados neste estudo corroboram importantes discussões no que se refere à criação de estratégias e condições apropriadas, não só para aumentar a frequência da prática de atividade física no lazer na população brasileira de uma forma geral, mas especialmente para a população que apresenta menor frequência desta prática, como as mulheres, os idosos e as pessoas de baixa escolaridade e condições socioeconômicas precárias. Esta reflexão deve perpassar pelos profissionais de saúde, gestores púbicos e sociedade civil e organizada.

Nesse sentido, programas populacionais e políticas de promoção da saúde voltados para incentivar a prática de atividade física na comunidade e nas escolas, assim como, políticas públicas na área do planejamento urbano e ambiente, são estratégias potencializadoras para aumentar os níveis de atividade física na população e para promoção de hábitos de vida mais saudáveis.

\section{Referências}

Booth, F. W., Roberts, C. K., Thyfault, J. P., Ruegsegger, G. N., \& Toedebusch, R. G. (2017). Role of Inactivity in Chronic Diseases: Evolutionary Insight and Pathophysiological Mechanisms. Physiol Rev. 97(4),1351-1402. https://doi.10.1152/physrev.00019.2016.

Bull, F., Goenka, S., Lambert, V. \& Pratt, M. (2017). Physical Activity for the Prevention of Cardiometabolic Disease. In: Prabhakaran D, Anand S, Gaziano TA, Mbanya JC, Wu Y, Nugent R, editors. Cardiovascular, Respiratory, and Related Disorders. (3a ed.) Washington (DC): The International Bank for Reconstruction and Development / The World Bank. 25. 10.1596/978-1-4648-0518-9_ch5

Bull, F. C., Al-Ansari, S. S., Biddle, S., Borodulin, K., Buman, M. P., Cardon, G., Carty, C., Chaput, J. P., Chastin, S., Chou, R., Dempsey, P. C., DiPietro, L., Ekelund, U., Firth, J., Friedenreich, C. M., Garcia, L., Gichu, M., Jago, R., Katzmarzyk, P. T., Lambert, E., ... Willumsen, J. F. (2020). World Health Organization 2020 guidelines on physical activity and sedentary behaviour. British journal of sports medicine, 54(24), 1451-1462. https://doi.org/10.1136/bjsports-2020-102955.

Carvalho, F. F. B. (2019). Recomendações de atividade física para a saúde (pública): reflexões em busca de novos horizontes. ABCS Health Sciences. 44 (2),327-345. https://dx.doi.org/10.7322/abcshs.v44i2.1199.

Chor, D., Cardoso, L. O., Nobre, A. A., Griep, R. H., Fonseca, M. J. M., Giatti, L., Bensenor, I., Molina, M. D. C., Aquino E. M. L., Diez-Roux, A., Pina, C. D., \& Santos, S. M. (2016). Association between perceived neighbourhood characteristics, physical activity and diet quality: results of the brazilian longitudinal study of adult health (elsa-brasil). Bmc Public Health. 16(1),1-11. https://dx.doi.10.1186/s12889-016-3447-5.

Dumith, S. C., Santos, M. N., Teixeira, L. O., Cazeiro, C. C., Mazza, S. E. I., \& Almeida A.C. (2016). Prática de atividade física entre jovens em município do semiárido no Brasil. Cien Saude Colet. 21(4),1083-1093. https://dx.doi.10.1590/1413-81232015214.18762015.

Guthold, R., Stevens, G. A., Riley, L. M., \& Bull, F. C. (2018). Worldwide trends in insufficient physical activity from 2001 to 2016 : a pooled analysis of 358 population-based surveys with 1.9 million participants. Lancet Global Health. 6(10),1077-1086. https://doi.10.1016/S2214-109X(18)30357-7.

Instituto Brasileiro de Geografia e Estatística - IBGE (2015). “A Síntese dos Indicadores Sociais 2018 - Uma Análise das Condições de Vida da População Brasileira". http://www.ibge.gov.br

Instituto Brasileiro de Geografia e Estatística - IBGE (2020). Pesquisa nacional de saúde: 2019: percepção do estado de saúde, estilos de vida, doenças crônicas e saúde bucal: Brasil e grandes regiões / IBGE, Coordenação de Trabalho e Rendimento. - Rio de Janeiro: IBGE. 113p. https://biblioteca.ibge.gov.br/visualizacao/livros/liv101748.pdf

Jones, S. A., Leeman, J., \& Evenson, K. R. (2020). Physical Activity Facilitators and Barriers Among Retired Women in North Carolina: A Qualitative Study. N C Med J. 81(5): 284-292. 10.18043/ncm.81.5.284

Ministério da Saúde (BR). (2012). Secretaria de Vigilância em Saúde. Secretaria de Gestão Estratégica e Participativa. Vigitel Brasil 2011: vigilância de fatores de risco e proteção para doenças crônicas por inquérito telefônico / Ministério da Saúde, Secretaria de Vigilância em Saúde, Secretaria de Gestão Estratégica e Participativa. - Brasília: Ministério da Saúde; 2012.

Moreno-Llamas, A., García-Mayor, J., \& De la Cruz-Sánchez, E. (2020). Physical activity barriers according to social stratification in Europe. Int J Public Health. 65(8),1477-1484. https://doi.10.1007/s00038-020-01488-y

Morris, K. A., Arundell, L., Cleland, V., \& Teychenne, M. (2020). Social ecological factors associated with physical activity and screen time amongst mothers from disadvantaged neighbourhoods over three years. The international journal of behavioral nutrition and physical activity, 17(1), 110. https://doi.org/10.1186/s12966-020-01015-5

Oliveira, A. J., Lopes, C. S., Rostila, M., Werneck, G. L., Griep, R. H., \& Ponce de Leon, A. C. (2014). Gender differences in social support and leisure-time physical activity. Rev Saúde Pública. 48(4), 602-612. https://dx.doi.10.1590/S0034-8910.2014048005183.

Patterson, R., McNamara, E., Tainio, M., Sá, T. H., Smith, A. D., Sharp, S. J., Edwards, P., Woodcock, J., Brage, S., \& Wijndaele, K. (2018). Sedentary behaviour and risk of all-cause, cardiovascular and cancer mortality, and incident type 2 diabetes: a systematic review and dose response meta-analysis. Eur $J$ Epidemiol. 33(9),811-829. https://doi.10.1007/s10654-018-0380-1. 
Research, Society and Development, v. 10, n. 11, e314101119560, 2021

(CC BY 4.0) | ISSN 2525-3409 | DOI: http://dx.doi.org/10.33448/rsd-v10i11.19560

Pitanga, F. J. G., Matos, S. M. A., Almeida, M. C., Griep, R. H., Viana, M. C., Melo, E. C.P., \& Aquino, E. (2018). Factors associated with sedentary behavior among ELSA-Brasil participants: ecological model. Rev Bras Ativ Fís Saúde. 23,1-8. https://dx.doi.10.12820/rbafs.23e0006.

Silva, C. L., Souza, M. F., Rossi Filho, S., Silva, L. F., \& Rigoni, A. C. C. (2017). Atividade física de lazer e saúde: uma revisão sistemática. Mudanças Psicologia da Saúde. 25(1),57-65. https://doi.org/10.15603/2176-1019/mud.v25n1p57-65.

Silva, D. A. S., Tremblay, M. S., Souza, M. F. M., Ribeiro, A. L. P., Cousin, E., Nascimento, B. R., Valença Neto, P. F., Naghavi, M., \& Malta, D. (2020). Physical inactivity as a risk factor for all-cause mortality in Brazil (1990-2017) Popul Health Metrics. 18(1),1-9. https://doi.org/10.1186/s12963-020-00214-3.

Villalobos, F., Vinuesa, A., Pedret, R., Reche, A., Domínguez, E., Arija, V., \& Equipo de investigación «Pas a Pas» (2019). Efecto de un Programa de actividad física sobre la autoestima en sujetos con enfermedades crónicas. Ensayo de intervención comunitaria. Atencion primaria, 51(4), 236-244. https://doi.org/10.1016/j.aprim.2017.11.011

Wendt, A., Carvalho, W. R. G., Silva, I. C. M., \& Mielke, G. I. (2019). Preferências de atividade física em adultos brasileiros: resultados da Pesquisa Nacional de Saúde. Rev Bras Ativ Fís Saúde. 24,1-9. https://dx.doi.10.12820/rbafs.24e0079.

Werneck, A. O., Baldew, S. S., Miranda, J. J., Díaz, A. O., Stubbs, B., \& Silva, D. R. (2019). On the behalf of the South American Physical Activity and Sedentary Behavior Network (SAPASEN) collaborators. Physical activity and sedentary behavior patterns and sociodemographic correlates in 116,982 adults from six South American countries: the South American physical activity and sedentary behavior network (SAPASEN). Int J Behav Nutr Phys Act. 16(1):68. $10.1186 / \mathrm{s} 12966-019-0839-9$.

World Health Organization (2018). Global action plan on physical activity 2018-2030: more active people for a healthier world. https://www.who.int/publications/i/item/9789241514187. ISBN 978-92-4-151418-7. 